\section{Personalized treatment of uveal melanoma}

Department of Molecular and Clinical Cancer Medicine, Ocular Oncology Service, Royal Liverpool University Hospital, Liverpool, UK

\section{Correspondence:}

B Damato, Department of Molecular and Clinical

Cancer Medicine, Ocular Oncology Service, Royal Liverpool University Hospital, Prescot Street, Liverpool L7 8XP, UK Tel: +44 (0)151706 3973; Fax: +44 (0)151 7065436. E-mail: Bertil@Damato.co.uk

Received: 8 October 2012 Accepted in revised form: 8 October 2012

Published online:

23 November 2012

Presented at the Cambridge Ophthalmological

Symposium, 14 September 2012.

\begin{abstract}
Personalized treatment of uveal melanoma involves the tailoring of all aspects of care to the condition, needs, wishes, and fears of the patient, taking account of the individual's circumstances. When selecting between radiotherapy, surgical resection, and phototherapy, or when deciding how best to combine these different therapeutic modalities, it is necessary to understand the patients utilities, with respect to tumour control, visual conservation, and preservation of the eye, so as to prioritize outcomes accordingly. For example, such considerations would influence the width of the safety margins when administering radiotherapy, according to whether the patient considers it more important to conserve vision or to guarantee tumour control. With 'suspicious naevi', the choice between observation, immediate treatment, and biopsy is complicated by the lack of adequate survival data on which to base rational decisions, making it necessary for both patient and doctor to accept uncertainty. Personalized care should involve close relatives, as appropriate. It must also adapt to changes in the patient's needs over time. Such personalized care demands the ability to respond to such needs and the sensitivity to identify these requirements in the first place. Personalized treatment enhances not only the patient's satisfaction but also the 'job satisfaction' of all members of the multidisciplinary team, improving quality of care.
\end{abstract}

Eye (2013) 27, 172-179; doi:10.1038/eye.2012.242; published online 23 November 2012

Keywords: uveal melanoma; treatment; melanoma

The term 'personalized treatment' can be defined as the tailoring of therapy to the needs,
B Damato and H Heimann

wishes, fears, and condition of the patient, also taking account of the individual's circumstances. In the context of holistic care, this also encompasses counselling, consent, and psychological support, which must be provided not only to the patient but also to the family.

Uveal melanomas can cause visual handicap, facial disfigurement, pain, and metastatic death. ${ }^{1}$ Many patients experience visual loss, caused by the tumour or its treatment, and some are restricted by such deficit. About a third of patients require enucleation. A very few patients develop painful complications, such as neovascular glaucoma. Almost $50 \%$ of patients develop metastatic disease, which is almost always fatal. ${ }^{2}$ Each patient responds to these threats in an individual way.

There is a wide range of therapeutic options. These include various forms of radiotherapy, phototherapy, and surgical resection, each with its own indications, contraindications, risks, and benefits. ${ }^{3}$ These modalities can be administered alone or in combination. Management is complicated by the rarity of this disease (ie, approximately 6 per million per year), making it necessary for patients to be treated at specialist adult ocular oncology centres, which in the United Kingdom are currently located in Glasgow, Liverpool, London, and Sheffield. Many patients are elderly, with the age at presentation peaking at 60 years. ${ }^{4}$ Comorbidity is therefore common.

The main therapeutic objectives, such as visual preservation and tumour control, often conflict with each other, with priorities varying greatly between patients. This difficult situation is compounded by the fact that decision making may be hampered by the lack of good evidence, making it necessary to 'act without facts' (ie, to gamble). For example, it is not known whether ocular treatment influences survival and if so in whom. ${ }^{5}$

Practitioners (and patients) vary greatly in their attitude to interacting with each other, some preferring the 'paternalistic' approach and 
others requiring a more consensual decision making. In theory, it is the patients who should choose their treatment, after being told all they need to know; in practice, however, it is difficult for them to comprehend the large amounts of information they are given and to provide fully informed consent in time for treatment the next day. Delaying treatment for days or weeks to give patients time to consider all options is usually not helpful, making it necessary to tailor the decision-making process to the individual. ${ }^{6}$

There is so much variation between ocular oncology centres that any attempt to provide an encyclopaedic overview would prevent an adequate discussion of the subject. The aim of this review is to describe how personalized care is provided to patients with uveal melanoma at our centre in Liverpool.

\section{Treatment planning}

\section{Management of pigmented tumours of indeterminate malignancy ('suspicious naevus')}

The first decision to be made is whether or not to treat the ocular tumour. If it is not possible to determine clinically whether this is a naevus or melanoma, it can be difficult to choose between observation, treatment, or biopsy (Figure 1a). Although observation is the most convenient choice, the risk of metastasis associated with delaying treatment is not known. A patient observed in Liverpool had a choroidal melanoma that suddenly grew after several years of apparent inactivity. ${ }^{7}$ Examination of the enucleated eye showed features suggesting late transformation from low-risk disomy 3 to highly lethal monosomy 3. The patient died of metastatic disease, which may have been prevented if she had been treated without delay. In many patients, early treatment would unnecessarily cause visual loss and perhaps other complications. Increasingly, this dilemma is resolved by tumour biopsy, which, however, is not without its risks (ie, haemorrhage, endophthalmitis, tumour seeding, and biopsy failure, with an inconclusive result). In theory, randomized trials of treatment versus observation of small, asymptomatic tumours should provide the answer; in practice, however, such studies would require thousands of patients and many years of follow-up, especially as large numbers of patients would decline an invitation to participate or they would drop out because their tumour becomes symptomatic or if they become concerned about metastatic risk. Our approach is to split one big decision into several small steps, guiding the patients through the various scenarios, describing what might happen and helping them to contemplate on how they might feel after each possible outcome. In most patients, the best way forward soon becomes obvious.
The minority who cannot immediately make up their mind are given a 4-month follow-up appointment, with the option to discuss matters with the specialist ocular oncology nurse at any time, and to let us know if they decide to have treatment so that this can be administered without delay. We also have a 'buddy' system so that patients can speak to volunteers who have previously been through a similar experience.

\section{Treatment of melanoma}

A review of 3680 patients undergoing eye-conserving therapy in Liverpool, between 1993 and 2012 indicates that $35 \%$ of patients underwent primary enucleation, the remainder having eye-conserving treatment consisting of plaque radiotherapy in $32 \%$, proton beam radiotherapy in $22 \%$, trans-scleral local resection in $7 \%$, endoresection in $3 \%$, and phototherapy in $1 \%$. After conservative therapy, local tumour control is achieved in $91 \%$, with $90 \%$ of all patients retaining the eye.

The choice of ocular treatment is determined by the chances of conserving the eye and useful vision; the risk of ocular and systemic complications; the visual needs; general health; utilities (ie, priorities); and the patient's ability or preparedness to attend the ocular oncology centre for treatment and follow-up.

Ocular factors The main ocular factors predicting loss of the eye are: tumour thickness and proximity to the optic disc. ${ }^{8}$ Other risk factors are large diameter; extensive involvement of the ciliary body, iris, or angle; diffuse growth pattern; and secondary effects such as neovascular glaucoma, total retinal detachment, and severe uveitis. Extraocular spread does not preclude conservative treatment if resectable or if it can be included in the proton beam field. ${ }^{9}$ The chances of conserving vision are diminished by proximity to the fovea or optic disc, a long history of visual loss caused by retinal detachment, and large tumour bulk.

Patients' ocular needs The patient's ocular needs are influenced by the visual acuity in the fellow eye and the patient's occupation. Some patients consider the eye to be useless without vision and others, such as teachers, are more concerned about retaining their own eye, even if this has no useful vision.

Patients' feelings The patients' feelings have a major role in decision making. Some patients decline radiotherapy because they cannot bear the thought of having a persistent tumour in the eye, despite reassurances that the chances of recurrence are minimal. When considering radiotherapy, some patients are happy to reduce safety margins, in the hope of conserving vision, whereas others 

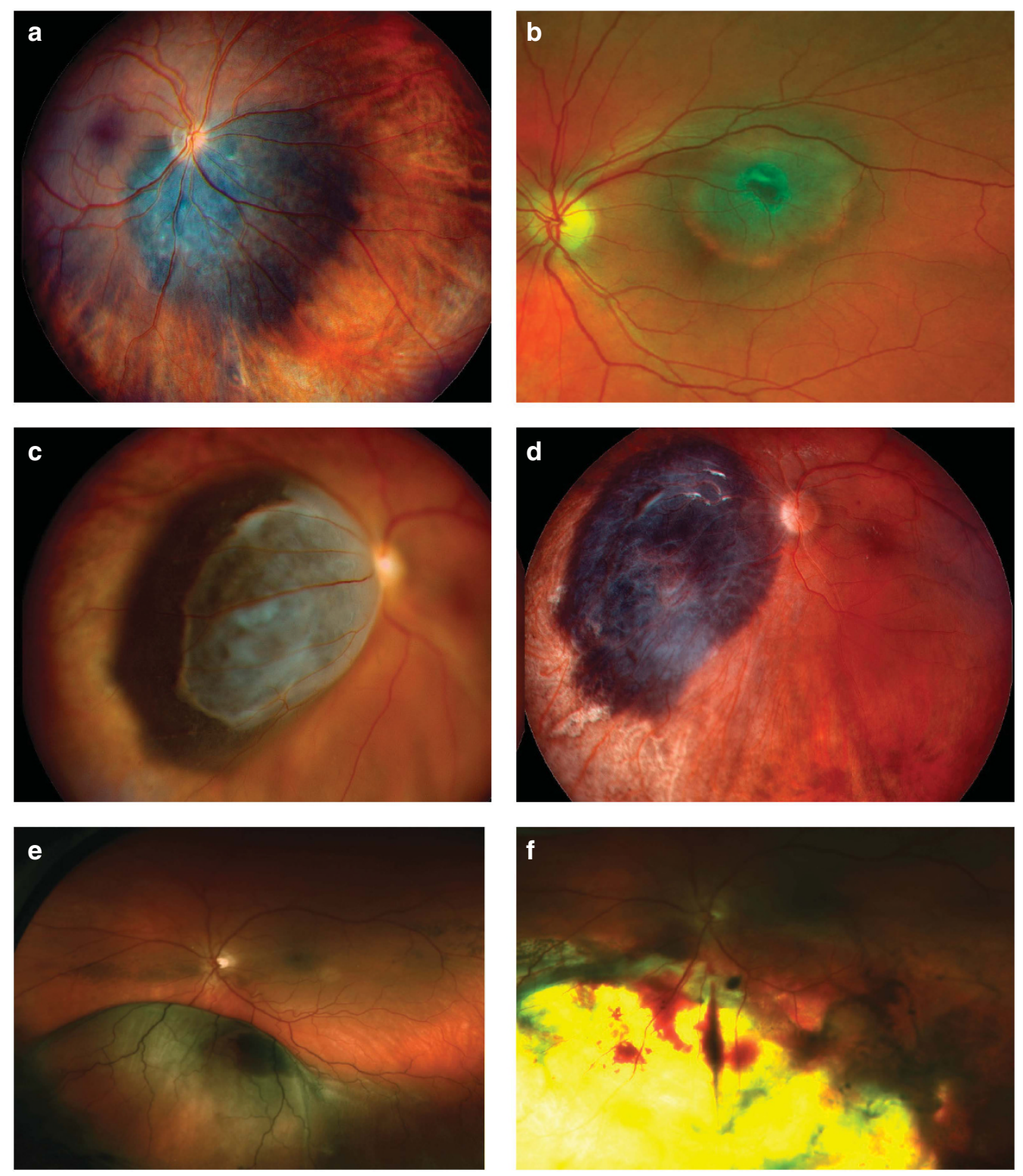

Figure 1 (a) A melanocytic tumour of indeterminate malignancy in the right eye of a 48-year-old woman. The choice of management included: observation, with an unknown risk of metastasis; proton beam radiotherapy, which would inevitably have caused visual loss; and diagnostic biopsy, with risks of retinal detachment, haemorrhage, infection, and failure. As the fellow eye was amblyopic, with visual acuity of $6 / 18$, the patient was observed. Thirteen years later, her condition was unchanged, with visual acuity of $6 / 6$. (b) Left eye of a 38-year-old woman with a melanocytic tumour of indeterminate malignancy measuring 5.9 mm basally with a thickness of $1.4 \mathrm{~mm}$. The patient was offered the choice between observation, immediate proton beam radiotherapy, and biopsy. She was unhappy about leaving a potentially malignant tumour untreated and did not wish to sacrifice vision unnecessarily if the tumour was benign. She therefore had a biopsy, which showed the lesion to be a spindle-cell melanoma. Proton beam radiotherapy was administered and 6 months later the visual acuity was 6/9. (c) Left eye of a 58-year-old woman with a juxtapapillary choroidal melanoma measuring $12.8 \mathrm{~mm}$ basally, with a thickness of $4.8 \mathrm{~mm}$. The choice of treatment included enucleation, proton beam radiotherapy, plaque radiotherapy, and endoresection. (d) The patient opted for proton beam radiotherapy, receiving this together with transpupillary thermotherapy, which induced resolution of the exudative retinal detachment. Five years later the patient was well, with visual acuity of 6/6. (e) Left eye of a 45-year-old man with an inferonasal choroidal melanoma measuring $16.4 \mathrm{~mm}$ basally with a thickness of $5.9 \mathrm{~mm}$, associated with an extensive exudative retinal detachment. The choice of treatment included ruthenium plaque radiotherapy, proton beam radiotherapy, trans-scleral local resection, and enucleation. (f) The patient underwent trans-scleral local resection because he needed good visual acuity to continue to work as a fireman. Four weeks post-operatively, the visual acuity had regained the pre-treatment level of $6 / 19$ and was improving. 
insist on the full treatment, even if this inevitably causes visual loss. When offered trans-scleral local resection, some patients baulk at the thought of hypotensive anaesthesia when they are informed about the possible complications (myocardial infarction and cerebrovascular accident), although these are exceedingly rare. Some patients decline endoresection when informed about other ophthalmologists' concerns about tumour seeding. Such fears are based on intuition rather than evidence as seeding is very rare, with an incidence of approximately $1 \% \cdot{ }^{10,11}$

Logistical considerations Most patients live far from the ocular oncology centre, and this unfortunately often influences the choice of treatment, especially if there are difficult domestic circumstances. Some patients opt for enucleation because they care for a sick relative or because their occupation prevents them from being absent for long periods (eg, farmers). Occasionally, these factors influence the choice between plaque and proton beam radiotherapy, the former being completed in a week and the latter requiring the patient first to have an operation for insertion of tantalum markers, then to attend Clatterbridge Centre for Oncology for treatment planning, a few weeks later, and then to return to that hospital a week or two later for the treatment itself.

Interactive decision making As mentioned before in this article, we prefer the consensual approach to treatment selection. We believe that patients find it easier to come to terms with any loss or inconvenience if they have a say in their treatment planning. For this reason, when the chances of conserving an eye are slim, we do not immediately say to the patient that the eye must be removed; instead, we describe all the complications that are likely to happen after local resection or radiotherapy, so that it is the patient who tells us that they consider enucleation to be the best treatment. Many patients find it difficult to take responsibility for such an important decision. We therefore relieve them of this burden as soon as possible by reassuring them that they have made what we consider to be the wisest decision (if this is indeed the case, which it usually is). Some patients, especially from overseas, expect paternalistic care and find it perplexing to travel a long distance for an expert opinion only to be told that it is up to them to decide what to do. Careful explanation is required if the patient's trust and confidence are to be maintained.

Therapeutic options With choroidal melanomas, the first choice of eye-conserving treatment in Liverpool is ruthenium plaque radiotherapy. ${ }^{12,13}$ This is because, unlike proton beam radiotherapy, there is no damage to the eyelids and the external eye and because, as mentioned above, it is completed within a week. It is also much less expensive (Table 1$){ }^{3}$ If the tumour extends close to the optic disc or fovea, the plaque is positioned so that its posterior edge is aligned with the posterior tumour margin. This is done with the patient's consent and using tools and techniques developed in Liverpool. ${ }^{14}$ In most patients, a minimum scleral dose of $350 \mathrm{~Gy}$ is delivered, so as to cause visible choroidal atrophy by 6 months; if this atrophy confirms that the plaque was positioned adequately, the patient can be discharged from our care, to be followed-up at the local hospital. If the patient is diabetic, then the minimum safe apex dose is given, delivering adjunctive transpupillary thermotherapy to prevent tumour recurrence and exudation. If the chances of local tumour recurrence are estimated to exceed $5 \%$, because the tumour has an irregular shape, extends close to optic disc, or has a thickness $>5 \mathrm{~mm}$, then another form of treatment is selected.

If ruthenium plaque radiotherapy is not appropriate, then the second choice of treatment is proton beam radiotherapy. ${ }^{15}$ This may be combined with transpupillary thermotherapy to reduce exudation. With superior tumours, proton beam radiotherapy is delivered through the closed eyelid to avoid lash loss and keratinization of the mucocutaneous junction at the lid margin, which causes painful keratitis. With bulky tumours, radiotherapy causes severe exudative retinal detachment, rubeosis, and neovascular glaucoma ('toxic tumour syndrome'), so that trans-scleral local resection is performed, if appropriate. ${ }^{16}$ Surgical excision is also preferred with medial tumours, because proton beam radiotherapy of such tumours tends to cause canalicular damage and intractable epiphora. When the tumour extends close to the optic disc or fovea, then the patient is offered proton beam radiotherapy with a notch designed to reduce the radiation delivered to these structures. With medial tumours approximating or involving the optic disc, the patient is offered endoresection, unless $>5$ clock hours of the optic disc are involved or unless the basal tumour diameter exceeds $10 \mathrm{~mm} \cdot{ }^{17,18}$ When the tumour is very small and thin, transpupillary thermotherapy or photodynamic therapy may be considered, provided that the patient accepts that such treatment may need to be followed by radiotherapy for persistent tumour. ${ }^{19}$ If none of these forms of conservative therapy is appropriate, then enucleation is advised.

For iris melanomas, our first choice of treatment is proton beam radiotherapy, because this is an outpatient procedure with acceptable morbidity. ${ }^{20}$ If the tumour is diffuse, then whole-anterior segment proton beam radiotherapy is delivered. ${ }^{21}$ Most patients will require cataract surgery and anti-glaucoma therapy, with some requiring medical treatment for limbal stem cell failure. 
Table 1 Treatment selection in Liverpool

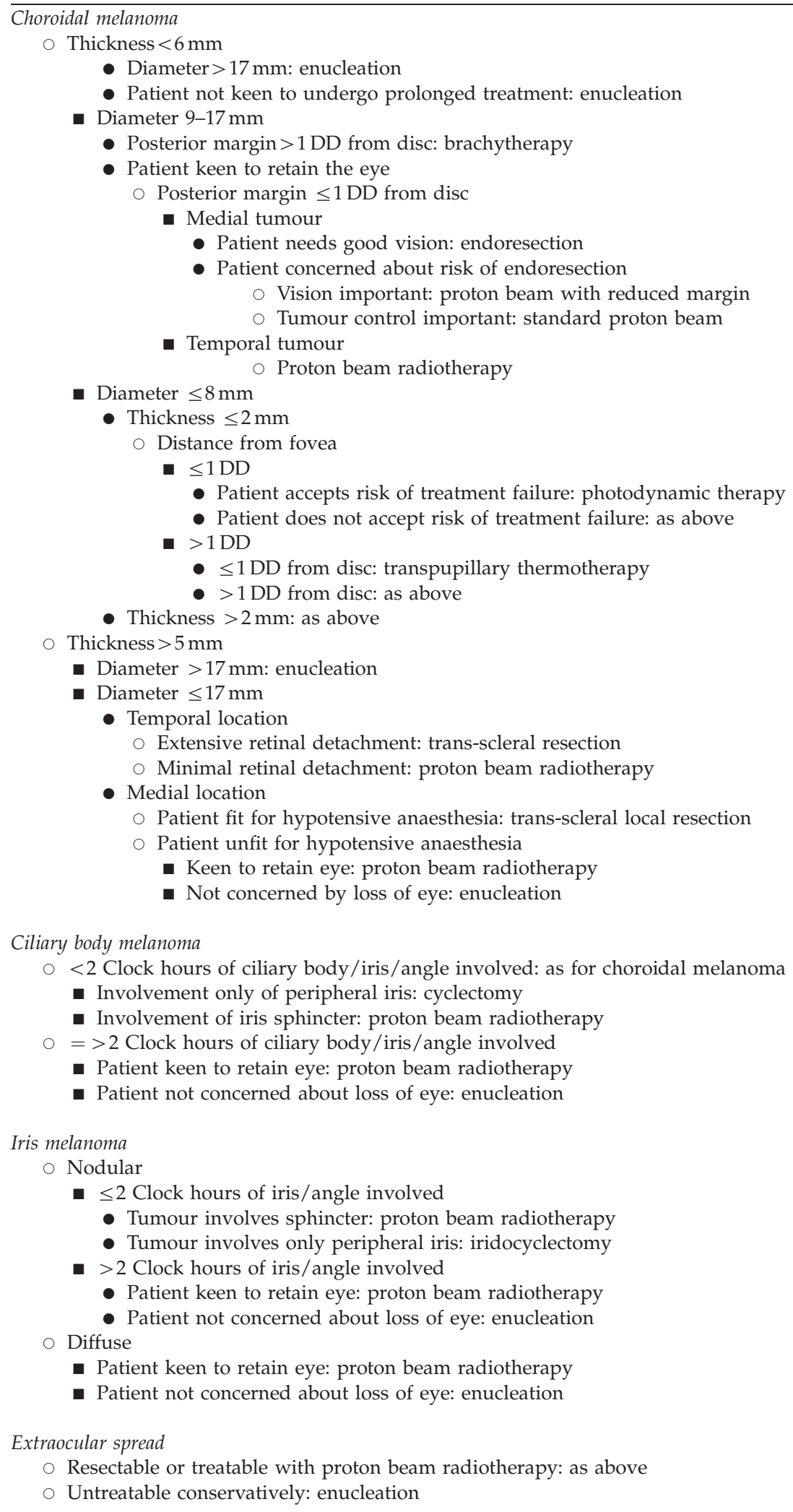


Small ciliary body tumours are surgically removed using techniques developed by the first author (BD) to conserve the iris sphincter. Such excision also provides ample tissue for diagnosis and prognostication. ${ }^{22}$

Rational treatment selection must, of course, be based on evidence, the most relevant data being those of the centre where the patient is treated. We rely heavily on our own customized ocular oncology database, with data entered prospectively by two full-time data managers / secretaries. Patients with ocular malignancy are reported to the NHS Cancer Registry, so that we are automatically notified of the date and cause of death of any of our cases from mainland Britain. All patients are invited to complete quality-of-life questionnaires 6 months after their initial treatment and then annually. By undertaking these measures, we are able to advise patients, not only on how likely they are to survive and retain the eye and vision but also on how they will actually feel.

\section{Estimating risk of metastatic disease}

Prognostication allows screening for metastatic disease to be targeted at high-risk individuals while avoiding expensive and stressful investigations in patients with little or no risk of metastasis. It also reduces anxiety, not only in patients with a favourable prognosis, as one would expect, but also in those with a high-risk of metastasis (at least in Britain). ${ }^{23,24}$ This is because patients feel empowered when they know their prognosis and when they can prepare themselves and their family for any eventuality. Most attend an oncologist for 6-monthly screening. Although the chances of prolonging life are slim, patients find such screening helpful because a normal liver scan reassures them that they are unlikely to develop symptomatic metastatic disease in the next 6 months. Our experience is that the most anxious and unhappy patients are those whose prognosis is uncertain. This is especially the case when they are denied the benefits of biopsy, because this is considered inappropriate (eg, small tumour size) or because the sample is inadequate for analysis.

Conventionally, survival is predicted according to the tumour size and extent, using methods such the TNM staging system (tumour, node, metastasis) of the American Joint Committee on Cancer. Such anatomical staging provides only a very crude estimate of survival probability. An online prognostic tool has been developed in Liverpool, which enhances reliability, making predictions relevant to individuals. ${ }^{25}$ This is achieved by integrating TNM stage with histological grade of malignancy and genetic tumour type. Metastatic disease occurs almost exclusively in patients whose tumour shows chromosome 3 loss and/or a class 2 gene expression profile. ${ }^{26,27}$ With such tumours, clinical tumour stage indicates how long the tumour and any metastases have been growing whereas histological grade of malignancy correlates with rate of tumour growth. Such personalized prognostication has profoundly influenced patient care, at least at our centre. ${ }^{24}$ For this reason, in Liverpool and a growing number of centres, patients undergoing radiotherapy are routinely offered a prognostic tumour biopsy (Figure 2).

Not all ocular oncologists are convinced about the benefits of prognostic biopsy. Some still feel that there is no point in performing risky and expensive tumour biopsy to predict metastatic disease that only rarely responds to treatment. In the United Kingdom, however, patients have formed an organization (ie, Ocumel UK) so that more are finding out how the care they received at their oncology centre compares with that provided elsewhere. We have heard of patients who have become upset because their ophthalmologist did not offer them the option of prognostic tumour biopsy. They feel let down because the 'informed consent' they provided was not as informed as they would have liked. This is a difficult and controversial area.

\section{Counselling}

Our approach is to discuss the advantages and disadvantages of each type of management so that patients can decide what best fits their own attitude to risk. Each new patient is given a CD-ROM or an audiocassette tape recording of the actual discussions to help them remember what was said. ${ }^{28}$ Three-dimensional photographs of their eye and a plastic model help them to visualize their tumour. Immediately after this explanation, they are seen by a specialist nurse, who ensures that they have an adequate understanding of their condition and its treatment. Patients also receive copies of the reports that are sent to their local ophthalmologist. Furthermore, they are able to get in touch with our specialist nurse by phone or e-mail at any time if they ever wish to discuss any aspect of their care. A full-time health psychologist screens all new patients for psychological morbidity, providing support as appropriate or organizing such care close to the patient's home. These measures all help to personalize care to each individual patient.

\section{Follow-up}

In Liverpool, the treatment of uveal melanoma is designed to minimize the need for patients to attend repeatedly for follow-up assessments unless they develop a complication. It is assumed that patients do not wish to travel far from home unless this is essential. Some, however, are keen to be followed-up at our ocular 
a

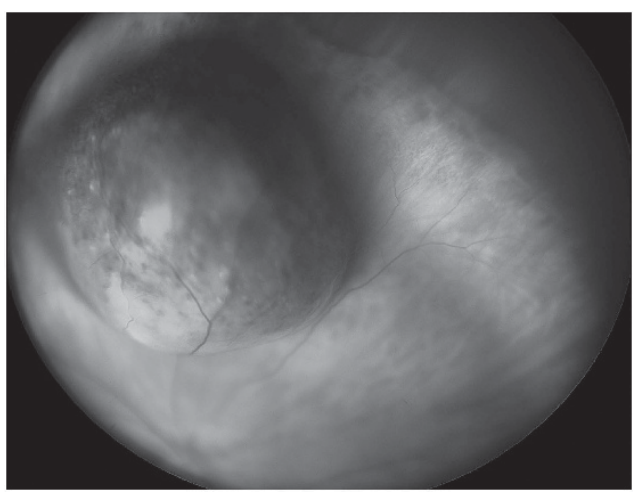

b

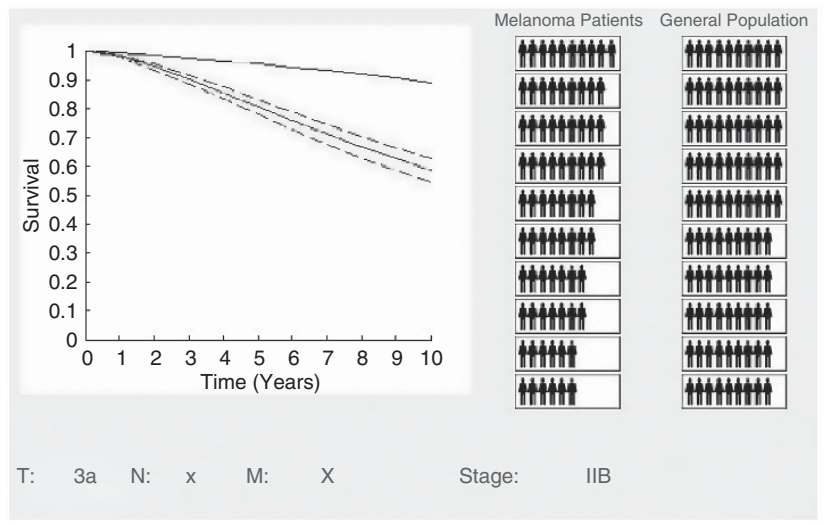

C

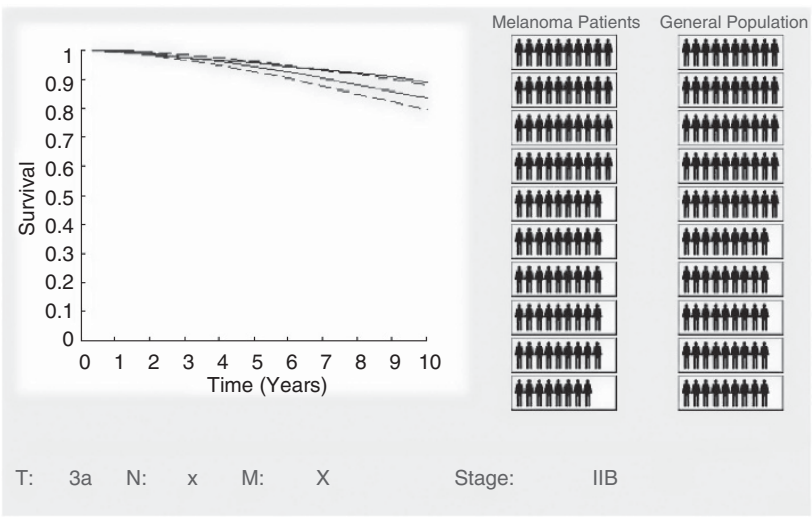

d

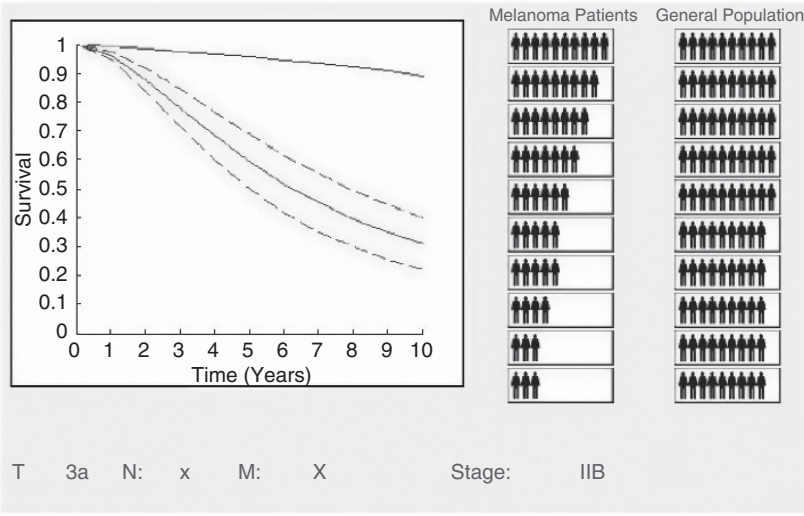

Figure 2 (a) Right eye of a 57-year-old man with a superior choroidal melanoma, which measured $15.2 \mathrm{~mm}$ basally with a thickness of $5.2 \mathrm{~mm}$ (ie, TNM stage IIB). The patient was treated with a $20 \mathrm{~mm}$ ruthenium-106 plaque. (b) The clinical features, considered alone, would have indicated a 30\% risk of metastatic death at 10 years. (c) The patient underwent a prognostic trans-retinal biopsy, which showed the tumour to be of mixed cell type, with no chromosome 3 loss. Multivariate analysis using our online predictor indicated the 10-year risk of metastasis to be only 5\%. (d) If this biopsy had shown chromosome 3 loss, this risk would have been 58\%. Almost 5 years later, the patient was alive with good local tumour control, no metastatic disease, and visual acuity 6/9.

oncology centre. If such patients do not need to see an ocular oncologist, because the risk of ocular complications is low, they are reviewed at a follow-up clinic run by specialist oncology nurses. ${ }^{29}$ Surveys using anonymous questionnaires have shown high levels of satisfaction among patients attending these clinics.

\section{Conclusions}

Personalized treatment of uveal melanoma yields high levels of patient satisfaction with the care they receive. It also improves the 'job satisfaction' of all members of the multidisciplinary team, enhancing motivation and efficiency, improving quality of care. Individualization of ocular treatment is possible only if an adequate selection of therapeutic modalities is available. Such a multimodality approach requires sufficient patient numbers, to achieve expertise with every kind of treatment and to maintain an adequate cost-benefit ratio.

Patients' needs tend to change over time and it is necessary to adapt to such change if personalized care is to be maintained. This requires not only an adequate responsiveness to each patient's requirements but also the sensitivity and empathy to recognize such needs in the first place.

\section{Conflict of interest}

The authors declare no conflict of interest.

\section{Acknowledgements}

We acknowledge the National Specialist Commissioning Group, which funded the Liverpool Ocular Oncology Service. We are also grateful to Dr Iwona RospondKubiak for assistance with the illustrations. 


\section{References}

1 Damato B. Progress in the management of patients with uveal melanoma. The 2012 Ashton Lecture. Eye (Lond) 2012; 26(9): 1157-1172.

2 Kujala E, Makitie T, Kivelä T. Very long-term prognosis of patients with malignant uveal melanoma. Invest Ophthalmol Vis Sci 2003; 44(11): 4651-4659.

3 Damato BE. Treatment selection for uveal melanoma. Dev Ophthalmol 2012; 49: 16-26.

4 Damato BE, Coupland SE. Differences in uveal melanomas between men and women from the British Isles. Eye (Lond) 2012; 26(2): 292-299.

5 Damato B. Does ocular treatment of uveal melanoma influence survival? Br I Cancer 2010; 103(3): 285-290

6 Bridson JD, Damato B. Consent to rapid treatment of eye tumours: is the waiting time too short at the Liverpool Ocular Oncology Centre? Clin Ethics 2009; 5: 86-94

7 Callejo SA, Dopierala J, Coupland SE, Damato B. Sudden growth of a choroidal melanoma and multiplex ligation-dependent probe amplification findings suggesting late transformation to monosomy 3 type. Arch Ophthalmol 2011; 129(7): 958-960.

8 Damato B, Lecuona K. Conservation of eyes with choroidal melanoma by a multimodality approach to treatment: an audit of 1632 patients. Ophthalmology 2004; 111(5): 977-983.

9 Muen WJ, Damato BE. Uveal malignant melanoma with extrascleral extension, treated with plaque radiotherapy. Eye (Lond) 2007; 21(2): 307-308.

10 Damato B. Choroidal melanoma endoresection, dandelions and allegory-based medicine. Br J Ophthalmol 2008; 92(8): 1013-1014.

11 Garcia-Arumi J, Zapata MA, Balaguer O, Fonollosa A, Boixadera A, Martinez-Castillo V. Endoresection in high posterior choroidal melanomas: long-term outcome. Br J Ophthalmol 2008; 92(8): 1040-1045.

12 Damato B, Patel I, Campbell IR, Mayles HM, Errington RD. Visual acuity after Ruthenium(106) brachytherapy of choroidal melanomas. Int J Radiat Oncol Biol Phys 2005; 63(2): 392-400.

13 Damato B, Patel I, Campbell IR, Mayles HM, Errington RD. Local tumor control after 106Ru brachytherapy of choroidal melanoma. Int J Radiat Oncol Biol Phys 2005; 63(2): 385-391.

14 Russo A, Laguardia M, Damato B. Eccentric ruthenium plaque radiotherapy of posterior choroidal melanoma. Graefe's Archive for Clinical and Experimental Ophthalmology 2012; 250(10): 1533-1540.

15 Damato B, Kacperek A, Chopra M, Campbell IR, Errington RD. Proton beam radiotherapy of choroidal melanoma: the Liverpool-Clatterbridge experience. Int J Radiat Oncol Biol Phys 2005; 62(5): 1405-1411.
16 Damato BE. Local resection of uveal melanoma. Dev Ophthalmol 2012; 49: 66-80.

17 Damato B, Groenewald C, McGalliard J, Wong D. Endoresection of choroidal melanoma. Br J Ophthalmol 1998; 82(3): 213-218.

18 Garcia-Arumi J, Sararols L, Martinez V, Corcostegui B. Vitreoretinal surgery and endoresection in high posterior choroidal melanomas. Retina 2001; 21(5): 445-452.

19 Shields CL, Shields JA, Cater J, Lois N, Edelstein C, Gunduz K et al. Transpupillary thermotherapy for choroidal melanoma: tumor control and visual results in 100 consecutive cases. Ophthalmology 1998; 105(4): 581-590.

20 Damato B, Kacperek A, Chopra M, Sheen MA, Campbell IR, Errington RD. Proton beam radiotherapy of iris melanoma. Int J Radiat Oncol Biol Phys 2005; 63(1): 109-115.

21 Tan AN, Hoevenaars JG, Webers CA, Damato B, Beckers HJ. Baerveldt implant for secondary glaucoma due to iris melanoma. Clin Ophthalmol 2010; 4: 407-409.

22 Damato EM, Damato B, Sibbring JS, Coupland SE. Ciliary body melanoma with partial deletion of chromosome 3 detected with multiplex ligation-dependent probe amplification. Graefe's Archive for Clinical and Experimental Ophthalmology 2008; 246(11): 1637-1640.

23 Cook SA, Damato B, Marshall E, Salmon P. Reconciling the principle of patient autonomy with the practice of informed consent: decision-making about prognostication in uveal melanoma. Health Expectations: An International Journal of Public Participation in Health Care and Health Policy 2011; 14(4): 383-396.

24 Cook SA, Damato B, Marshall E, Salmon P. Psychological aspects of cytogenetic testing of uveal melanoma: preliminary findings and directions for future research. Eye (Lond) 2009; 23(3): 581-585.

25 Damato B, Eleuteri A, Taktak AF, Coupland SE. Estimating prognosis for survival after treatment of choroidal melanoma. Prog Retinal Eye Res 2011; 30(5): 285-295.

26 Damato B, Dopierala JA, Coupland SE. Genotypic profiling of 452 choroidal melanomas with multiplex ligationdependent probe amplification. Clin Cancer Res 2010; 16(24): 6083-6092.

27 Onken MD, Worley LA, Char DH, Augsburger JJ, Correa $\mathrm{ZM}$, Nudleman E et al. Collaborative ocular oncology group report number 1: prospective validation of a multi-gene prognostic assay in uveal melanoma. Ophthalmology 2012; 119(8): 1596-1603.

28 Ah-Fat FG, Sharma MC, Damato BE. Taping outpatient consultations: a survey of attitudes and responses of adult patients with ocular malignancy. Eye (Lond) 1998; 12(Pt 5): 789-791.

29 Sandinha T, Hebbar G, Kenawy N, Hope-Stone L, Damato B. A nurse-led ocular oncology clinic in Liverpool: results of a 6-month trial. Eye (Lond) 2012; 26(7): 937-943. 